Functional Analysis and Its Applications, Vol. 40, No. 4, pp. 273-284, 2006

Translated from Funktsional'nyi Analiz i Ego Prilozheniya, Vol. 40, No. 4, pp. 33-48, 2006

Original Russian Text Copyright (c) by D. W. H. Gillam and V. P. Gurarii

\title{
On Functions Uniquely Determined by Their Asymptotic Expansion
}

\section{W. H. Gillam and V. P. Gurarii}

Received April 7, 2006

In memory of Boris Yakovlevich Levin

ABstRACt. We present a maximal class of analytic functions. The elements of this class are uniquely determined by their asymptotic expansions. We also discuss the possibility of recovery of a function from the coefficients of its asymptotic series. In particular, we consider the problem of recovering by using Borel summation. The last published result in this direction was obtained by Alan Sokal in 1980, but his paper well known to physicists (in quantum field theory) seems to have remained unnoticed by mathematicians.

KEY WORDS: Watson's uniqueness theorem, Gevrey expansions, Laplace transforms in complex domain, differential equations in complex domain.

\section{Introduction}

In 1912, Watson published the following result [17, Sec. 8, Theorem V].

Watson's uniqueness theorem. Let $\left\{p_{0}, p_{1}, \ldots\right\}$ be a given sequence of complex numbers, and let $P(z)$ be a function satisfying the conditions

(i) $P(z)$ is analytic and single-valued in the sector $S(\alpha, \beta)$,

(ii) for all $z \in S(\alpha, \beta)$ and every $n, n=0,1, \ldots$,

$$
\left|\mathscr{R}_{n, P}(z)\right| \leqslant M \frac{n !}{a^{n}|z|^{n+1}},
$$

where the positive constants $M$ and a do not depend on $z$ and $n$ but may depend on $P(z)$.

If the opening of the sector $S(\alpha, \beta)$ satisfies the condition $\beta-\alpha>\pi$, then the function $P(z)$ is uniquely determined by conditions (i) and (ii), i.e., two functions $P_{1}(z)$ and $P_{2}(z)$ satisfying conditions (i) and (ii) with the same sequence $\left\{p_{0}, p_{1}, \ldots\right\}$ must coincide on $S(\alpha, \beta)$.

Here, $S(\alpha, \beta)$ stands for the sector $S(\alpha, \beta)=\{z \in \mathbb{C}: 0<|z|<\infty, \alpha<\arg z<\beta\}$; the number $\beta-\alpha$ is called the opening of the sector.

For a given function $P(z)$ analytic in $S(\alpha, \beta)$ and a given sequence of complex numbers $\left\{p_{0}, p_{1}, \ldots\right\}$, we set

$$
\mathscr{R}_{n, P}(z)=P(z)-\sum_{k=0}^{n-1} \frac{p_{k}}{z^{k+1}} .
$$

This expression is called the remainder.

Since the set of functions satisfying conditions (i) and (ii) is linear, it suffices to prove Watson's uniqueness theorem for the case where $\left\{p_{0}, p_{1}, \ldots\right\}=\{0,0, \ldots\}$. In this situation, we have

$$
|P(z)| \leqslant M \frac{n !}{a^{n}|z|^{n+1}}, \quad n=0,1, \ldots, z \in S(\alpha, \beta) .
$$

For a fixed $z,|z|>1 / a$, let us minimize the right-hand side of (2) with respect to $n$ to obtain the inequality

$$
|P(z)| \leqslant M_{a} e^{-a|z|}
$$

where $M_{a}=4 M \sqrt{2 \pi} a$. To complete the proof and thus to show that $(3) \Longrightarrow P(z) \equiv 0$, we need the following lemma. 
Lemma 1. Suppose that $P(z)$ is analytic in a sector $S(\alpha, \beta)$ with opening greater than $\pi$ and satisfies estimate (3) in this sector. Then $P(z) \equiv 0$.

For a proof based on the Phragmén-Lindelöf theorem, see [8, Sec. 8.11].

If the opening of the sector $S(\alpha, \beta)$ satisfies the condition $\beta-\alpha<\pi$, then the above uniqueness result is indisputably false. Indeed, given $\delta, 0<\delta<\pi / 2$, every function $P(z)$ of the form $P(z)=$ $\varphi(z) e^{-z} / z$, where $\varphi(z)$ is analytic and bounded in the sector $S=S(-\pi / 2+\delta, \pi / 2-\delta)$, satisfies condition (1) with $M=\sup _{S}|\varphi(z)|, a=\sin \delta$, and $p_{k}=0$ for $k=0,1, \ldots$. This can easily be shown using the elementary inequalities

$$
e^{-|z|}<\frac{n !}{|z|^{n}}, \quad n=0,1, \ldots
$$

Thus, the set of functions which satisfy conditions (i) and (ii) of Watson's theorem with $\left\{p_{0}, p_{1}, \ldots\right\}$ $=\{0,0, \ldots\}$ is rather large.

The two opposite cases, $\beta-\alpha>\pi$ and $\beta-\alpha<\pi$, discussed above lead us to regard the situation with $\beta-\alpha=\pi$ as the critical case and the value $\pi$ as the critical value of the opening $\beta-\alpha$.

In fact, the assertion of Watson's uniqueness theorem remains true for this critical case as well. Although Hardy's argument in [8, Sec. 8.11] is not applicable here, it is possible to use the Phragmén-Lindelöf theorem to prove the uniqueness, and the proof can be made even simpler. Assume that inequality (3) is valid in the sector $S(-\pi / 2, \pi / 2)$. Given $b, b>0$, consider the function $P_{b}(z)=P(z) e^{b z}$ inside the sector $S(-\arctan (b / a), \arctan (b / a))$. On the boundary of this sector we have $a|\operatorname{Im} z|-b \operatorname{Re} z=0$, and therefore $\left|P_{b}(z)\right| \leqslant M_{a}$. The standard Phragmén-Lindelöf theorem yields the same estimate inside the sector as well. Thus, for $z>0$, we have $|P(z)| \leqslant M_{a} e^{-b z}$. Since $b$ does not depend on $z$, we let $b$ tend to $+\infty$ to obtain $P(z) \equiv 0$.

\section{Gevrey Expansions of Order $k$}

Watson's uniqueness theorem can be restated for any sector $S(\alpha, \beta)$ of the Riemann surface of $\log z$ with opening greater than or equal to $\pi / k$, where $k$ is a positive number. For this, condition (ii) must be replaced by

$$
\left|\mathscr{R}_{n, P}(z)\right| \leqslant M \frac{(n !)^{1 / k}}{(k a|z|)^{n+1}}, \quad n=0,1, \ldots
$$

If the opening of the sector $S(\alpha, \beta)$ satisfies the condition $\beta-\alpha \geqslant \pi / k$, then the function $P(z)$ is uniquely determined by the coefficients $\left\{p_{0}, p_{1}, \ldots\right\}$.

This result is derived using (4) to obtain, in place of (3), the inequality $|P(z)| \leqslant M_{a} e^{-a|z|^{k}}$, which can be transformed to (3) using the map $\zeta=z^{k}$.

Comparing again the two opposite cases $\beta-\alpha>\pi / k$ and $\beta-\alpha<\pi / k$, we say that a sector $S(\alpha, \beta)$ is critical if its opening $\beta-\alpha$ is equal to $\pi / k$.

Watson's theory, based on estimate (1), was further developed by Gevrey [5] (1918) with application to the theory of partial differential equations and, in particular, to the heat conduction equation and by F. Nevanlinna [9] (1919) whose results will be discussed below.

Watson's uniqueness theorem was extended by Carleman [3] to a more general situation. Carleman replaced $n$ ! in estimates (1) by a sequence of positive numbers $m_{n}$ and, under certain regularity conditions on the growth of $m_{n}$ as $n \rightarrow \infty$, gave necessary and sufficient conditions for uniqueness.

Expansions satisfying conditions similar to (4) are known as Gevrey expansions of order $k$ (see [5], [11], and [10]).

Since the beginning of the previous century, it has been known that sectors with opening $\pi / k$ play a special role in the asymptotic theory of systems of linear differential equations having an irregular singular point of Poincaré rank $k$ in the complex plane. In recent times it has been found that the Poincaré asymptotic expansions associated with regular solutions of such systems are in fact Gevrey expansions (see [10]). This property of asymptotic expansions turned out to be true 
not only for linear systems but also for a much larger class of analytic differential equations. This advancement in the theory of analytic differential equations is based on the following definition.

Definition. Let $P(z)$ be analytic and single-valued in a sector $S(\alpha, \beta)$ in the $z$-plane and let $\sum_{j=0}^{\infty} p_{j} / z^{j+1}$ be a formal power series with complex coefficients. Then this series is called the Gevrey asymptotic expansion of order $k, k>0$, for $P(z)$ in $S(\alpha, \beta)$ if for each proper subsector $S^{\prime}$ of $S(\alpha, \beta), \overline{S^{\prime}} \subset S(\alpha, \beta)$, there exist positive constants $M\left(S^{\prime}\right)$ and $a\left(S^{\prime}\right)$ such that the following system of relations is satisfied:

$$
\left|\mathscr{R}_{n, P}(z)\right| \leqslant M\left(S^{\prime}\right) \frac{(n !)^{1 / k}}{\left(k a\left(S^{\prime}\right)\right)^{n}|z|^{n+1}}, \quad z \in S^{\prime} .
$$

\section{The Main Theorem}

We develop Watson's result in the following direction. We consider an analytic function $P(z)$ which admits a Gevrey asymptotic expansion in a critical sector $S$ with opening equal to $\pi$, and our aim is to clarify under what conditions on $M\left(S^{\prime}\right)$ and $a\left(S^{\prime}\right)$, where $S^{\prime}$ is a proper subsector of $S$, the function possesses the uniqueness property. The most significant parameter defining the subsector $S^{\prime}$ inside $S$ is the angular deviation of its boundary from that of $S$, which we denote by $\delta$. For simplicity, we assume that $k=1$ and $S=S(-\pi / 2, \pi / 2)$. It then suffices to consider only subsectors of the form $S^{\prime}=S(-\pi / 2+\delta, \pi / 2-\delta)$, where $0<\delta<\pi / 2$. We shall denote the functions $M\left(S^{\prime}\right)$ and $a\left(S^{\prime}\right)$ by $M(\delta)$ and $a(\delta)$ and assume without loss of generality that $\log M(\delta)>1$.

The following generalization of Watson's uniqueness theorem for the critical sector $S(-\pi / 2, \pi / 2)$ is true.

Theorem 1. Assume that $P(z)$ admits a Gevrey expansion of order 1 in $S(-\pi / 2, \pi / 2)$. If $M(\delta)$ and $a(\delta)$ satisfy the conditions

$$
\int_{0}^{\pi / 2} \log \log M(\delta) d \delta<\infty
$$

and

$$
\frac{\delta}{a(\delta)} \rightarrow 0 \quad \text { as } \delta \rightarrow 0
$$

then $P(z)$ is uniquely determined by the coefficients of its Gevrey expansion.

It is possible to prove that if one of conditions (6) or (7) is violated, then (generally) the uniqueness property fails to hold.

Remark 1. In the situations in which $P(z)$ and $\widehat{P}(z), \widehat{P}(z)=\sum_{j=0}^{\infty} p_{j} / z^{j+1}$, are solutions (regular and formal, respectively) of a given analytic differential equation, the cases

$$
M(\delta)=M \exp \left(b / \delta^{\gamma}\right)
$$

and

$$
M(\delta)=M \exp (b / \delta),
$$

where the positive numbers $M, b$, and $\gamma$ do not depend on $\delta$, are of special interest.

\section{Stirling's Formula}

Analytic functions satisfying (1), (4) or (5) have long been known in analysis.

We recall an example based on Stirling's formula for Euler's gamma function, $\Gamma(z)$, which is analytic in the entire complex plane except at the points $z=0,-1, \ldots$ on the negative half-axis, where it has simple poles. In the sector $S(-\pi / 2, \pi / 2)$, the function $\Gamma(z)$ has the following integral representation:

$$
\Gamma(z)=\int_{0}^{\infty} t^{z-1} e^{-t} d t
$$


By Stirling's formula, we have

$$
\ln \Gamma(z)=\left(z-\frac{1}{2}\right) \ln z-z+\frac{1}{2} \ln 2 \pi+o(1), \quad z \rightarrow+\infty .
$$

We consider the so-called Binet function

$$
P(z)=\ln \Gamma(z)-\left(z-\frac{1}{2}\right) \ln z+z-\frac{1}{2} \ln 2 \pi
$$

and associate with this function a sequence $\left\{p_{0}, p_{1}, \ldots\right\}$ such that relations (1) are valid for some $M$ and $a$.

According to the first Binet formula, we have, for $z \in S(-\pi / 2, \pi / 2)$,

$$
P(z)=\int_{0}^{\infty} e^{-z t} F(t) d t
$$

where

$$
F(t)=\frac{1}{t}\left(\frac{1}{2}-\frac{1}{t}+\frac{1}{e^{t}-1}\right) .
$$

Since $F(t)$ is analytic in the disc $|t|<2 \pi$, it can be represented inside the disc by its Taylor series $\sum_{k=0}^{\infty} f_{k} t^{k}$. The substitution of this series for $F(t)$ into (11) yields

$$
\widehat{P}(z)=\sum_{k=0}^{\infty} \frac{p_{k}}{z^{k+1}}, \quad p_{k}=f_{k} k !
$$

Since $F(t)$ is an even function, $F(-t)=F(t)$, it readily follows that $p_{2 k-1}=0$ for $k=1,2, \ldots$, and a more detailed analysis shows that

$$
p_{2 k-2}=\frac{B_{2 k}}{2 k(2 k-1)}, \quad k=1,2, \ldots
$$

Here $B_{2 k}, k=0,1, \ldots$, are the Bernoulli numbers, which are defined as the coefficients of the Taylor series

$$
\frac{t}{e^{t}-1}=1-\frac{t}{2}+\sum_{k=2}^{\infty} B_{2 k} \frac{t^{2 k}}{(2 k) !} .
$$

For example, see formulas 23.1.1-23.1.3 and 6.1.42 in [1]. Thus, $\widehat{P}(z)$ can be rewritten in the form

$$
\widehat{P}(z)=\sum_{k=1}^{\infty} \frac{B_{2 k}}{2 k(2 k-1) z^{2 k-1}}
$$

known as Stirling's series*.

Using (11), one can show that $\widehat{P}(z)$ is the Gevrey expansion for $P(z)$ in $S(-\pi / 2, \pi / 2)$. Indeed, for $z \in S(-\pi / 2, \pi / 2)$, the estimates

$$
\begin{aligned}
|P(z)| & \leqslant K(z) \frac{\left|B_{2}\right|}{2|z|}, \\
\left|P(z)-\sum_{k=1}^{n} \frac{p_{2 k-2}}{z^{2 k-1}}\right| & \leqslant K(z) \frac{\left|B_{2(n+1)}\right|}{(2 n+2)(2 n+1)|z|^{2 n+1}}, \quad n=1,2, \ldots,
\end{aligned}
$$

are valid, where

$$
K(z)=\max _{u \geqslant 0}\left|\frac{z^{2}}{u^{2}+z^{2}}\right| .
$$

(For example, see formula 6.1.42 in [1].)

${ }^{*}$ A similar expression for Stirling's series is given in [19], but the notation $B_{k}$ used there refers to $(-1)^{k+1} B_{2 k}$, where $B_{2 k}$ is defined as above in (13). 
In particular, formula 23.1.15 in [1] yields the asymptotic behavior for the Bernoulli numbers

$$
B_{2 n+2}=(-1)^{n+2} \frac{2(2 n+2) !}{(2 \pi)^{2 n+2}}(1+o(1)), \quad k \rightarrow \infty,
$$

and it follows that (15) is of the form given in (1) with $a=2 \pi$. Thus, the Stirling series (14) is the Gevrey expansion for $P(z)$ in the sector $S(-\pi / 2, \pi / 2)$.

Remark 2. The importance of Stirling's example for this subject lies in the following observations:

(i) Using (16), it can be proved easily that estimate (15) ensures uniqueness in the sector $S(-\pi / 2, \pi / 2)$ with opening $\pi$ despite the fact that if $z$ belongs to the boundary of the sector, then the value of $K(z)$ is equal to $\infty$. Since the function $K(z)$ in (15) can be rewritten as

$$
K(z)= \begin{cases}1 & \text { for }-\pi / 4<\arg z<\pi / 4, \\ 1 / \sin (2|\arg z|) & \text { for } \pi / 4 \leqslant|\arg z|<\pi / 2,\end{cases}
$$

it follows that the Binet function given by (10)) satisfies all the conditions of Theorem 1 with $M(\delta)=1 / \delta$.

(ii) Substitution of $n_{\mathrm{opt}}(|z|)=[\pi|z|-1]$ for $n$ into inequality $(15)^{*}$ results in

$$
\left|P(z)-\sum_{k=1}^{[\pi|z|-1]} \frac{p_{2 k-1}}{z^{2 k-1}}\right| \leqslant K(z) \frac{2 \sqrt{2 \pi|z|}}{2 \pi|z|-1} e^{-2 \pi|z|} .
$$

For a given fixed $z \in S(-\pi / 4, \pi / 4)$, estimate (18) provides the best possible accuracy if $P(z)$ is replaced by the optimal finite sum of Stirling's series, and it follows from formula (17) that, for $|z|>1$, the error is less than

$$
M e^{-a|z|}
$$

where $M=0.94891$ and $a=2 \pi$.

(iii) It also follows from (11) and (12) that $\widehat{P}(z)$ is a Gevrey expansion of $P(z)$ in the whole sector $S(-\pi, \pi)$. However, estimate (1) with $a=2 \pi$ is valid only in $S(-\pi / 2, \pi / 2)$. It can be shown that, in a larger sector $S(-\pi / 2-\varepsilon, \pi / 2+\varepsilon)$, estimates similar to (1) are valid, but the constant $a$ in these estimates depends on $\varepsilon, a(\varepsilon)=2 \pi \cos \varepsilon$.

In principle, it is possible to derive a sufficient condition for uniqueness following, for example, the technique of Ahlfors' distortion theorem in [2] and its complement and refinement by Warschawski [16]. We present a proof which, firstly, is based on a simpler argument and, secondly, allows us to reveal the role of each of conditions (6) and (7) more precisely.

\section{The Main Lemma}

Theorem 1 is derived from the following generalization of Lemma 1.

Lemma 2. Assume that $P(z)$ is holomorphic in $S(-\pi / 2, \pi / 2)$. Furthermore, assume that, for every $\delta, 0<\delta<\pi / 2$, and for all $z, z \in S(-\pi / 2+\delta, \pi / 2-\delta)$, the function $P(z)$ satisfies the following estimate:

$$
|P(z)| \leqslant M(\delta) e^{-a(\delta)|z|}
$$

where $M(\delta)$ with $\log M(\delta)>1$ and $a(\delta), \delta \in(0, \pi / 2)$, satisfy conditions (6) and (7). Then

$$
P(z) \equiv 0 \text {. }
$$

Proof. Unfortunately, we cannot integrate the expression $e^{z t} P(z)$ neither along the imaginary axis nor along a line parallel to it. This obstacle is quite typical of such situations, and we shall show a way in which it can be overcome.

${ }^{*}$ This value of $n, n=n_{\mathrm{opt}}(|z|)$, can be obtained by minimizing the expression on the right-hand side of (15) for a given $|z|$. 
Given $\theta,-\pi / 2<\theta<\pi / 2$, introduce the Laplace transform

$$
F_{\theta}(t)=\int_{l_{\theta}} e^{z t} P(z) d z
$$

where $l_{\theta}=\{z: \arg z=\theta\}$.

In what follows we shall show that

(i) the function $F_{\theta}(t)$ can be continued analytically throughout the complex plane as an entire function $F(t)$ which does not depend on $\theta$,

(ii) the function $F(t)$ is bounded outside any sector $S_{\delta}, 0<\delta<\pi / 2$, with angle $2 \delta$, which has its apex at the point $a(\delta) /(2 \sin \delta)$, contains the interval $(a(\delta) /(2 \sin \delta),+\infty)$, and is symmetric with respect to the real axis,

(iii) on the boundary $\partial S_{\delta}$ of the sector $S_{\delta}$, we have $|F(t)| \leqslant 2 M(\delta) / a(\delta)$,

(iv) the function $F(t)$ satisfies all the conditions of Carleman's theorem, which we state below and whose application implies the identity $F(t) \equiv 0$.

Remark 3. V. Havin, to whom we showed our result, called our attention to the following fact. If one assumes that $M(\delta)$ in (20) satisfies the stronger condition (9) and that $a(\delta)$ is constant, $a(\delta)=a$, then, for any $c, 0<c<a$, there exists an $h>0$ and an $M_{h}>0$ such that

$$
|P(h+i y)|<M_{h} e^{-c|y|} .
$$

In this case, by Lemma $1, P(z) \equiv 0$.

To prove inequality (22), one needs only to note that if $z=x+i y$ and $x /|y|=\tan \delta$, then, using (9), inequality (20) can be rewritten in the form

$$
|P(z)|<M \exp \left(\frac{b}{\arctan (x /|y|)}\right) e^{-a|z|} .
$$

In turn, for $|y| \gg x>0,(23)$ implies the inequality

$$
|P(z)|<M^{\prime} \exp \left(\frac{b|y|}{x}\right) e^{-a|y|}
$$

for some constant $M^{\prime}>0$. From this inequality, after an appropriate choice of $x=h$, for example $h=b /(a-c),(22)$ follows immediately.

Unfortunately, this simple argument does not cover the case of faster growth of $M(\delta)$, for example, as in condition (8).

We now return to the proof.

5.1. Region of analyticity of $\boldsymbol{F}_{\boldsymbol{\theta}}(\boldsymbol{t})$. Everywhere in the proof we use the symbol $a$ for the function $a(\delta)$ except for the cases where we wish to emphasize the dependence on $\delta$. To simplify the proof, we also assume without loss of generality that $a(\delta)$ is a decreasing function of $\delta$. To find the region of analyticity of $F_{\theta}(t)$ we set

$$
\delta= \begin{cases}\pi / 2-\theta & \text { for } \theta>0 \\ \pi / 2+\theta & \text { for } \theta<0\end{cases}
$$

and derive the following estimate from (20) and (21):

$$
\left|F_{\theta}(t)\right| \leqslant M(\delta) \int_{l_{\theta}}\left|e^{z t}\right| e^{-a|z|} d|z| .
$$

Since

$$
\left|e^{z t}\right|=e^{|z|(\sigma \cos \theta-\tau \sin \theta)}, \quad t=\sigma+i \tau,
$$

integral (21) is absolutely convergent in the half-plane

$$
\Pi_{\theta, a}=\{t \in \mathbb{C}: \sigma \cos \theta-\tau \sin \theta<a\},
$$

and thus $F_{\theta}(t)$ is holomorphic there. In fact, if, say, $0<\theta<\pi / 2$, then the line

$$
L_{\theta, a}=\{t \in \mathbb{C}: \sigma \cos \theta-\tau \sin \theta=a\}
$$


divides the $t$-plane into two half-planes, and $\Pi_{\theta, a}$ is the half-plane containing the origin $t=0$, see Fig. 1.

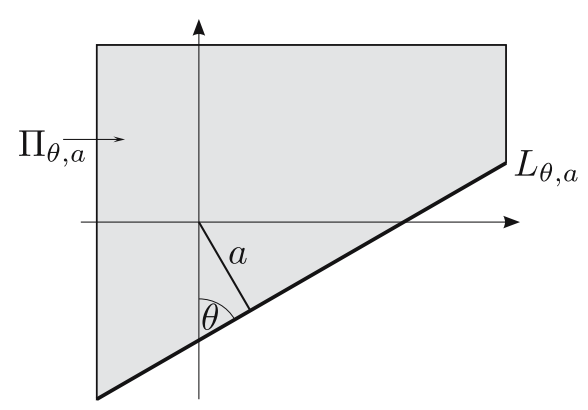

Fig. 1. The line $L_{\theta, a}$ and the half-plane $\Pi_{\theta, a}$

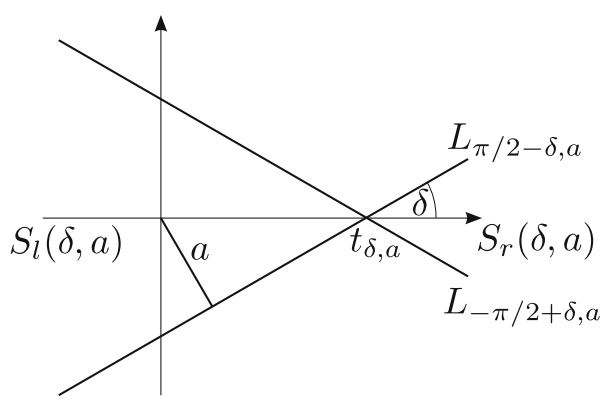

Fig. 2. The lines $L_{\mp \pi / 2 \pm \delta, a}$ and the sectors $S_{l}(\delta, a)$ and $S_{r}(\delta, a)$

Furthermore, for $t \in \Pi_{\theta, a},(24)$ and (25) imply the estimate

$$
\left|F_{\theta}(t)\right| \leqslant M(\delta) \frac{1}{a-(\sigma \cos \theta-\tau \sin \theta)} .
$$

In what follows, we shall show that all functions $F_{\theta}(t),|\theta|<\pi / 2$, are in fact elements of a single analytic function $F(t)$ which does not depend on $\theta$.

5.2. Relationship between $\boldsymbol{F}_{\boldsymbol{\theta}}(\boldsymbol{t})$ and $\boldsymbol{F}_{-\boldsymbol{\theta}}(\boldsymbol{t})$. Given $\delta, 0<\delta<\pi / 2$, consider the pair of functions $F_{\pi / 2-\delta}(t)$ and $F_{-\pi / 2+\delta}(t)$ of the form (21), which are holomorphic in the half-planes $\Pi_{\pi / 2-\delta, a}$ and $\Pi_{-\pi / 2+\delta, a}$, respectively,

$$
\begin{aligned}
\Pi_{\pi / 2-\delta, a} & =\{t \in \mathbb{C}: \sigma \sin \delta-\tau \cos \delta<a\}, \\
\Pi_{-\pi / 2+\delta, a} & =\{t \in \mathbb{C}: \sigma \sin \delta+\tau \cos \delta<a\} .
\end{aligned}
$$

These half-planes are symmetric with respect to the real axis in the $t$-plane.

Figure 2 shows the two lines $L_{\pi / 2-\delta, a}$ and $L_{-\pi / 2+\delta, a}$ intersecting the positive axis in the $t$-plane at the point

$$
t_{\delta, a}=\frac{a(\delta)}{\sin \delta}
$$

at angles $\delta$ and $-\delta$, respectively. These lines define two sectors, $S_{l}(\delta, a)$ (the left-hand sector) and $S_{r}(\delta, a)$ (the right-hand sector), both with apex at $t_{\delta, a}$ and with angle $2 \delta$.

Set

$$
S_{1}(\delta, a)=\Pi_{\pi / 2-\delta, a} \cap \Pi_{-\pi / 2+\delta, a} \quad \text { and } \quad S_{2}(\delta, a)=\Pi_{\pi / 2-\delta, a} \cup \Pi_{-\pi / 2+\delta, a} .
$$

The left-hand sector $S_{l}(\delta, a)$ is exactly $S_{1}(\delta, a)$, and the closure of the right-hand sector $S_{r}(\delta, a)$ is the complement of $S_{2}(\delta, a)$. (See Figs. 3 and 4.)

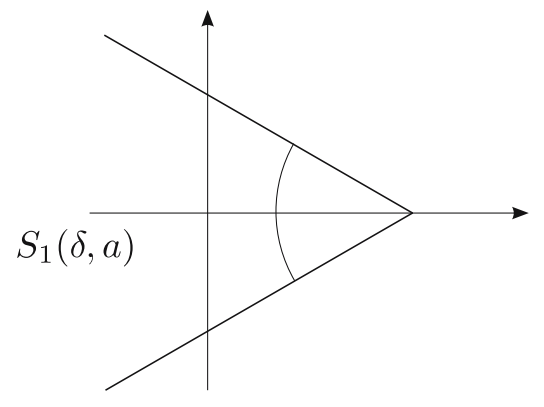

Fig. 3. The sector $S_{1}(\delta, a)$

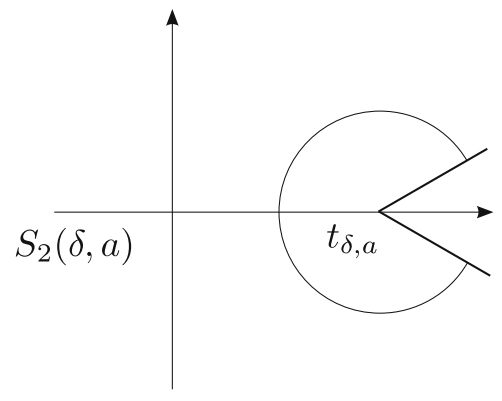

Fig. 4. The sector $S_{2}(\delta, a)$

Using representation (21) and Cauchy's theorem, we obtain

$$
F_{\pi / 2-\delta}(t)=F_{-\pi / 2+\delta}(t), \quad t \in S_{1}(\delta, a)
$$


Thus, $F_{\pi / 2-\delta}(t)$ and $F_{-\pi / 2+\delta}(t)$ are in fact elements of a function $F(t, \delta)$,

$$
F(t, \delta)= \begin{cases}F_{\pi / 2-\delta}(t) & \text { for } t \in \Pi_{\pi / 2-\delta, a}, \\ F_{-\pi / 2+\delta}(t) & \text { for } t \in \Pi_{-\pi / 2+\delta, a}\end{cases}
$$

and this function is holomorphic in $S_{2}(\delta, a)$.

5.3. The maximal region of analyticity. We need the following lemma.

Lemma 3. The functions $F(t, \delta)$ given by (30) are elements of a single analytic function $F(t)$ which does not depend on $\delta$ and which can be continued as an entire function throughout the complex $t$-plane.

Proof. Consider $\delta^{\prime}>\delta^{\prime \prime}>0$ and the corresponding functions $F\left(t, \delta^{\prime}\right)$ and $F\left(t, \delta^{\prime \prime}\right)$.

Since the function $a(\delta)$ is decreasing on the interval $(0, \pi / 2)$, we have

$$
S_{r}\left(\delta^{\prime}, a\right) \supset S_{r}\left(\delta^{\prime \prime}, a\right),
$$

and, consequently,

$$
\mathbb{C} \backslash S_{r}\left(\delta^{\prime}, a\right) \subset \mathbb{C} \backslash S_{r}\left(\delta^{\prime \prime}, a\right) .
$$

Using the same argument as above, we conclude that

$$
F\left(t, \delta^{\prime}\right)=F\left(t, \delta^{\prime \prime}\right), \quad t \in \mathbb{C} \backslash S_{r}\left(\delta^{\prime}, a\right),
$$

and therefore $F\left(t, \delta^{\prime \prime}\right)$ can be regarded as an analytic continuation of $F\left(t, \delta^{\prime}\right)$ to the larger region $\mathbb{C} \backslash S_{r}\left(\delta^{\prime \prime}, a\right)$.

By condition (7) of Theorem 1, expression (29) for $t_{\delta, a}$ shows that $t_{\delta, a} \rightarrow \infty$ as $\delta \rightarrow 0$, whence

$$
\bigcap_{0<\delta<\pi / 2} S_{r}\left(\delta^{\prime}, a\right)=\varnothing .
$$

Hence, the functions $F(t, \delta), 0<\delta<\pi / 2$, are elements of a single entire function $F(t)$, and thus assertion (i) has been verified.

5.4. Estimates for $\boldsymbol{F}(\boldsymbol{t})$ in the $\boldsymbol{t}$-plane. Using estimate (28) for the region

$$
\bar{\Pi}_{\theta, a / 2}=\Pi_{\theta, a / 2} \cup L_{\theta, a / 2},
$$

where $\Pi_{\theta, a / 2}$ and $L_{\theta, a / 2}$ are defined by (26) and (27), respectively, with $a$ replaced by $a / 2$, we conclude that

$$
\left|F_{\theta}(t)\right| \leqslant \frac{2 M(\delta)}{a}, \quad t \in \bar{\Pi}_{\theta, a / 2} .
$$

Hence, it follows from (30) that

$$
|F(t, \delta)| \leqslant \frac{2 M(\delta)}{a}, \quad t \in S_{2}(\delta, a / 2),
$$

and the same estimate is true for the function $F(t)$ in (i).

Now, in the sector $S_{2}(\delta, a / 2)$, the function $F(t, \delta)$ and, consequently, the function $F(t)$ are bounded, which confirms (ii). Moreover, it follows from (31) that $F(t)$ satisfies the estimate

$$
|F(t)| \leqslant \frac{2 M(\delta)}{a(\delta)}
$$

in the sector $S_{2}(\delta, a / 2)$, and hence on its boundary as well. This proves (iii).

Using (7) once again, we derive the following estimate from inequality (32):

$$
\left|F\left(|t| e^{ \pm i \delta}\right)\right|<\frac{2 M(\delta)}{\delta}
$$

this estimate is valid for sufficiently small $\delta$ and shows that the conditions of the following theorem by Carleman are satisfied. 
5.5. Carleman's Theorem [4]. Let $M(\varphi)$ be a positive (finite or infinite) function on $(0,2 \pi)$ such that $\log M(\varphi)>1$ and the integral

$$
\int_{0}^{2 \pi} \log \log M(\varphi) d \varphi
$$

is finite. Then every entire function $f(z)$ satisfying the inequality

$$
|f(z)|<M(\varphi), \quad \varphi=\arg z, 0<\varphi<2 \pi
$$

is identically constant.*

Thus, $F(t)=C$ for some constant $C$. To derive (iv), it remains to note that, since, for every $\delta$, we have

$$
F(t, \delta) \rightarrow 0 \text { as } t \rightarrow-\infty,
$$

it follows that the same relation is valid for $F(t)$, and thus $F(t) \equiv 0$. This proves (iv), whence $P(z) \equiv 0$ follows.

Remark 4. Carleman's result was further developed by N. Levinson and N. Sjoberg (for details, see the survey by M. Sodin [14]) who obtained the most general result from which, in particular, an extension of Carleman's theorem follows where the sector is replaced by a half-strip. Note, however, that, unlike Levinson's theorem, Carleman's theorem does not require any regularity of the majorant $M$. We believe that this extension may be useful in further investigation of the recovery problem, which we discuss below.

Watson's uniqueness theorem indicates the possibility of recovering a function $P(z)$ satisfying conditions (i) and (ii) of the theorem from the formal power series $\widehat{P}(z)$. This possibility can be realized using the Borel summation method. We refer to the corresponding result as Watson's recovery theorem. The proof of this theorem was presented by Watson in [17, Sec. 9]. Also see [18, p. 68 , formulas (20)-(22)] and [8, Sec. 8.11].

\section{Watson's Recovery Theorem}

Watson's recovery theorem. Assume that the function $P(z)$ satisfies conditions (i) and (ii) of Watson's uniqueness theorem in a sector $S(-\pi / 2-\varepsilon, \pi / 2+\varepsilon)$ for some $\varepsilon, 0<\varepsilon<\pi / 2$.

We set

$$
\widehat{F}(t)=\sum_{n=0}^{\infty} \frac{p_{n}}{n !} t^{n},
$$

which is the result of the Borel summation of $\widehat{P}(z)$. Then

(i) series (33) is absolutely convergent and represents an analytic function $F(t)$ in the disk $D_{a}$ with radius $a$ and center at the origin in the t-plane;

(ii) the function $F(t)$ can be continued analytically from the disk $D_{a}$ to the region

$$
D_{a, \varepsilon}=D_{a} \cup\{t \in \mathbb{C}:|\arg t|<\varepsilon\},
$$

see Fig. 5,

(iii) the function $P(z)$ can be represented as the Laplace transform of $F(t)$,

$$
P(z)=\int_{0}^{+\infty} F(t) e^{-z t} d t
$$

where the integral is absolutely convergent for $z \in S(-\pi / 2, \pi / 2)$.

*If the function $M(\varphi)$ satisfies the stronger condition (8), then this fact follows immediately from the PhragménLindelöf theorem. 


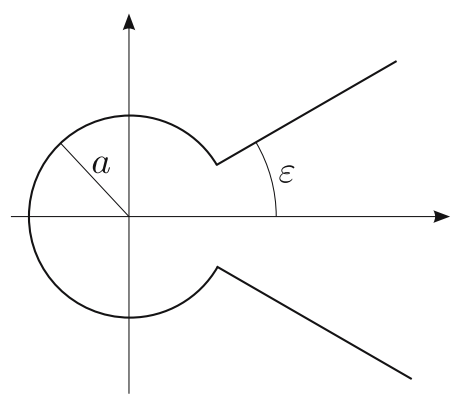

Fig. 5. The region $D_{a, \varepsilon}$

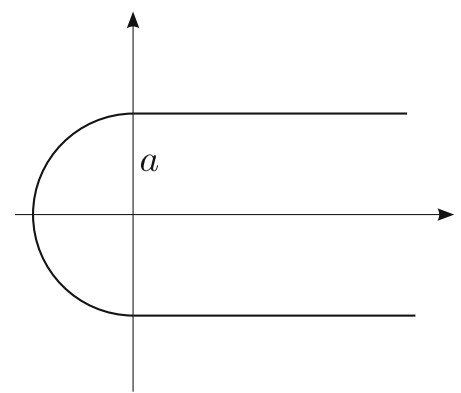

Fig. 6. The region $\mathscr{D}_{a}$

Two problems immediately arise here. (a) What happens to the region of analyticity (34) of $F(t)$ as $\varepsilon \rightarrow 0$ ? (b) If $P(z)$ satisfies (1) in $S(-\pi / 2-\varepsilon, \pi / 2+\varepsilon)$ for some fixed $a$ and $\varepsilon$, can part (ii) in the conclusion of Watson's recovery theorem be improved?

Question (a) was answered by F. Nevanlinna in [9] (1919) who dealt in fact with a more general case of Gevrey expansions of an arbitrary order $k$. However, the principal difficulty lies precisely in question (a). Curiously, Hardy in his book [8] on divergent series makes no reference to this improvement of Watson's result for the half-plane.

The improvement of Watson's recovery theorem (problem (b)) was not obtained until 1980 when A. D. Sokal discovered it and applied it to the perturbation expansion in $\phi_{2}^{4}$-quantum field theory, see [15]. Sokal's improvement gives a necessary and sufficient characterization for a large class of Borel summable functions.

However, one of the first reappearances of Watson's theorems occurred ten years earlier in connection with the theory of anharmonic oscillators, see [7].

Our Theorem 1 suggests a similar result for a function $P(z)$ satisfying the conditions of Theorem 1 in the critical sector $S(-\pi / 2, \pi / 2)$ with $M(\delta)$ and $a(\delta)$ satisfying (6) and (7), respectively.

Consider the simplest case in which $M(\delta)$ and $a(\delta)$ are positive constants. As was shown earlier, Watson's uniqueness theorem extends to this case, and this suggests the existence of the corresponding recovery theorem for $P(z)$.

Let us introduce the following region in the complex plane $\mathbb{C}$ :

$$
\mathscr{D}_{a}=\{t \in \mathbb{C}: \operatorname{dist}(t,(0,+\infty)) \leqslant a\},
$$

see Fig. 6 . This region is completely determined by the position of an arbitrary point of its boundary.

We shall refer to it as the determinative region.

Nevanlinna's recovery theorem. Assume that the function $P(z)$ satisfies conditions (i) and (ii) of Watson's uniqueness theorem in the right half-plane of the z-plane. Then $F(t)$ given by (33) can be continued analytically from the disk $D_{a}$ to the region $\mathscr{D}_{a}$ in the complex $t$-plane as a bounded function, the function $P(z)$ can be represented as the Laplace transform of $F(t)$ in formula (35), and the integral is absolutely convergent for $z \in S(-\pi / 2, \pi / 2)$.

Sokal [15] states without proof the following converse of Nevanlinna's theorem.*

Theorem. Given an $a>0$, let $F(t)$ be a bounded and analytic function in the region $\mathscr{D}_{a}$ of the complex t-plane with Taylor series $\sum_{k=0}^{\infty} f_{k} t^{k}$. Let $P(z)$ be its Laplace transform. Then $P(z)$ is holomorphic in the right half-plane of the complex $z$-plane, and $\sum_{k=0}^{\infty} f_{k} k ! / z^{k+1}$ serves as the Poincaré expansion of $P(z)$ satisfying estimate (1).

Moreover, Sokal showed how the assertion of Watson's recovery theorem can be improved using Nevanlinna's theorem, and the proof is quite simple. Indeed, assuming that the conditions of Watson's recovery theorem are satisfied, let us apply Nevanlinna's theorem to each subsector of $S(-\pi / 2-\varepsilon, \pi / 2+\varepsilon)$ with opening $\pi$. It follows that the function $F(t)$ given by (35) is holomorphic

${ }^{*}$ Sokal presents in fact a formally more general result considering, along with the right half-plane, its translations of the form $\operatorname{Re} z>b$, where $b$ is real. 
in the region $\mathscr{D}_{a, \theta}=e^{-i \theta} \mathscr{D}_{a},|\theta|<\varepsilon$, and thus, $F(t)$ is holomorphic in the region

$$
\mathscr{D}_{a}(\varepsilon)=\bigcup \mathscr{D}_{a, \theta} \text {. }
$$

Region (36) is bounded by the two tangents to the circle $\partial D_{a}$ at angles $\pm \varepsilon$ to the positive axis of the $t$-plane and also by the outer arc of the circle joining these tangents. (See Fig. 7.)

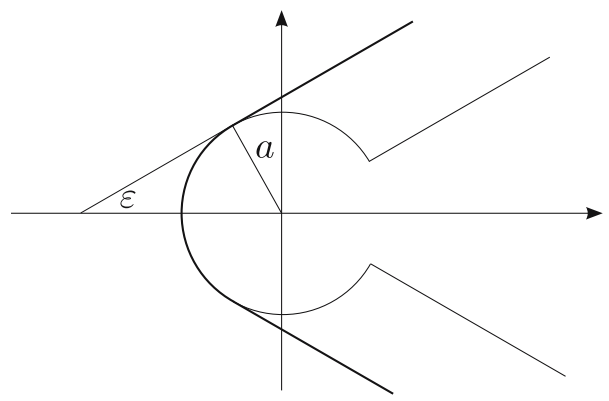

Fig. 7. The region $\mathscr{D}_{a}(\varepsilon)$

Remark 5. Let us try to apply Sokal's theorem to the situation in Sec. 4 . Note that the determinative region of the function $F(t)$ in $(12)$ is $\mathscr{D}_{2 \pi}$. Since $F(t)$ has a pole at the point $t=2 \pi i$ on the boundary of $\mathscr{D}_{2 \pi}$, this function does not satisfy the conditions of Sokal's theorem. Thus, the problem arises of how to modify Sokal's theorem to make it applicable to unbounded functions. For Stirling's example, this obstacle can be immediately overcome in the following way. Introduce the functions $F_{0}(t)=1 /\left(t^{2}+4 \pi^{2}\right)$ and $F_{1}(t)=F(t)-F_{0}(t)$. The determinative regions for these functions are $\mathscr{D}_{2 \pi}$ and $\mathscr{D}_{4 \pi}$, respectively. For the associated function $P_{0}(z)$ the optimal estimate for the remainder can be evaluated directly to obtain a result close to that in (18), and with the same function $K(z)$. As for the contribution of the associated function $P_{1}(z)$ to (18), using Sokal's theorem in the region $\mathscr{D}_{(4 \pi-\varepsilon)}$ yields a correction of order $O\left(e^{-(4 \pi-\varepsilon)|z|}\right)$, where $\varepsilon$ is small, and this result could be sharpened using a further improvement of Sokal's theorem.

Our aim is to extend these results to more general uniqueness classes of functions for which $M(\delta)$ is unbounded. We conjecture that it is still possible to recover $P(z)$ satisfying the conditions of Theorem 1 from its Gevrey expansion $\widehat{P}(z)$ using, as above, the Borel summation method if (6) is replaced by the stronger condition

$$
M(\delta)<M \exp (b / \delta) .
$$

It seems that, for classes of functions satisfying the conditions of Theorem 1 when the growth of $M(\delta)$ is faster than that indicated in (9), one should look for an alternative summation method.

For further discussion and extension of the results of Nevanlinna and Sokal, see [6].

\section{Conclusion}

Two points have motivated this work. The first is the discovery by Ramis and Sibuya [12], [13] that, roughly speaking, if a formal power series satisfies an analytic (not necessarily linear) differential equation, then there exists a $k>0$ such that, for each sector with (critical) opening $\pi / k$, there is a regular solution of the equation for which the formal solution is a Gevrey expansion of order $k$. Moreover, they proved the uniqueness of such a solution, and this is what led to a reexamination of Watson's theorem for critical openings of the sector. If $P(z)$ is the regular solution in the Ramis-Sibuya theorem, then we believe that $M(\delta)$ satisfies (8) and, probably, the stronger condition (9). However, this is an open question. The second point relates to the calculation of the best possible estimate for the deviation of a function from finite sums of its Gevrey expansion. This can be done if one obtains the best possible estimates for $M(\delta)$ and $a\left(\delta^{-1}\right)$ analogous to those in (19) for Stirling's example.

Acknowledgment. The authors wish to express their gratitude to Nick Garnham and Peter Jones and to the School of Mathematical Sciences of Swinburne University of Technology for 
encouragement and support and also to Victor Katsnelson and David Lucy for numerous helpful discussions. The authors are especially grateful to Iossif Ostrovski whose corrections and suggestions allowed us to improve significantly the original version of this paper.

The second author is grateful to the Weizmann Institute of Science, Israel, for support under the Rosi and Max Varon Visiting Professorship program.

\section{References}

[1] M. J. Abramowitz, I. A. Stegun, Handbook of Mathematical Functions, with Formulas, Graphs, and Mathematical Tables, Dover Publications, New York, 1965.

[2] L. V. Ahlfors, Conformal Invariants. Topic in Geometric Function Theory, McGraw-Hill, New York-Düsseldorf-Johannesburg, 1973.

[3] T. Carleman, Les fonctions quasy-analytiques, Gauthier-Villars, Paris, 1926.

[4] T. Carleman, "Extension d'un théorème de Liouville," Acta Math., 48 (1926), 363-366; in: Édition Complète des Articles de Torsten Carleman, Inst. Math. Mittag-Leffler, Malmö, 1960, 271-274.

[5] M. Gevrey, "Sur la nature analytique des solutions des équations aux dérivées partielles," Ann. Sci. Écol. Norm. Sup. (3), 35 (1918), 129-190.

[6] D. W. H. Gillam, V. Gurarii, "On the Watson-Nevanlinna recovering theorem," in preparation.

[7] S. Graffi, V. Grecchi, B. Simon, "Borel summability: Application to the anharmonic oscillator," Phys. Lett., 32B (1970), 631-634.

[8] G. H. Hardy, Divergent Series, Oxford: at the Clarendon Press, 1949.

[9] F. Nevanlinna, "Zur Theorie der Asymptotischen Potenzreihen," Ann. Acad. Sci. Fenn. Ser. A, XII:3 (1919), 1-81.

[10] J.-P. Ramis, "Théorèmes d'indices Gevrey pour les équations différentielles ordinaires," Mem. Amer. Math. Soc., 48:296 (1984).

[11] J.-P. Ramis, "Séries divergentes et théories asymptotiques," Bull. Soc. Math. France, 121 (1993).

[12] J.-P. Ramis, Y. Sibuya, "Hukuhara Domain and Fundamental Existence and Uniqueness Theorems for Asymptotic Solutions of Gevrey Type," Asymptotic Analysis, 2 (1989), 39-94.

[13] J.-P. Ramis, Y. Sibuya, "A new proof of multisummability of formal solutions of non linear meromorphic differential equations," Ann. Inst. Fourier, 44:3 (1994), 811-848.

[14] M. Sodin, "Lars Ahlfors' thesis," in: Lectures in Memory of Lars Ahlfors, Israel Math. Conf. Proc. (Haifa, 1996), vol. 14, Bar-Ilan Univ., Ramat Gan, 2000, 113-134.

[15] A. D. Sokal, "An improvement of Watson's theorem on Borel summability," J. Math. Phys., $\mathbf{2 1 : 2}(1980), 261-263$.

[16] S. Warschawski, "On conformal mapping of infinite strips," Trans. Amer. Math. Soc., 51 (1942), 280-335.

[17] G. N. Watson, "A theory of asymptotic series," Phil. Trans. Roy. Soc. London, Ser. A, 211 (1912), 279-313.

[18] G. N. Watson, "The transformation of an asymptotic series into a convergent series of inverse factorial," Circ. Math. Palermo Rend., 34 (1912), 41-88.

[19] E. T. Whittaker, G. N. Watson, A Course of Modern Analysis, Cambridge Univ. Press, Cambridge, 1996.

Faculty of Engineering and Industrial Sciences

SWINBURNe University of TECHNOLOGY

e-mail: dgillam@swin.edu.au

School of Mathematical Sciences

Monash UNIVERSITY

e-mail: vladimir.gurarii@sci.monash.edu.au 